\title{
The TW Hya Rosetta Stone Project. I. Radial and Vertical Distributions of DCN and $\mathrm{DCO}^{+}$
}

\author{
Karin I. Öberg $^{1}$ (D), L. Ilsedore Cleeves ${ }^{2}$ (D), Jennifer B. Bergner ${ }^{3}$ (D), Joseph Cavanaro ${ }^{1}$, Richard Teague ${ }^{1}$ (D), Jane Huang ${ }^{1,4}$ (D), \\ Ryan A. Loomis $^{5}$ (D), Edwin A. Bergin ${ }^{6}$ (ID), Geoffrey A. Blake ${ }^{7}$ (D), Jenny Calahan ${ }^{6}$ (D), Paolo Cazzoletti ${ }^{8}$, Viviana Veloso Guzmán ${ }^{9}$, \\ Michiel R. Hogerheijde ${ }^{8,10}$ (D) , Mihkel Kama ${ }^{11}$ (D), Jeroen Terwisscha van Scheltinga ${ }^{8,12}$ (D), Chunhua Qi ${ }^{1}$ (D), Ewine van Dishoeck ${ }^{8}$ (iD), \\ Catherine Walsh ${ }^{13}$ (D), and David J. Wilner ${ }^{1}$ (i) \\ ${ }^{1}$ Harvard-Smithsonian Center for Astrophysics, 60 Garden Street, Cambridge, MA 02138, USA; koberg@cfa.harvard.edu \\ ${ }^{2}$ Department of Astronomy, University of Virginia, Charlottesville, VA 22904, USA \\ ${ }^{3}$ NASA Sagan Fellow, University of Chicago Department of the Geophysical Sciences, Chicago, IL 60637, USA \\ ${ }^{4}$ NHFP Sagan Fellow, Department of Astronomy, University of Michigan, 323 West Hall, 1085 S. University Avenue, Ann Arbor, MI 48109, USA \\ ${ }^{5}$ National Radio Astronomy Observatory, Charlottesville, VA 22903, USA \\ ${ }^{6}$ Department of Astronomy, University of Michigan, 1085 S. University Avenue, Ann Arbor, MI 48109, USA \\ ${ }^{7}$ Division of Geological \& Planetary Sciences, California Institute of Technology, Pasadena CA 91125, USA \\ ${ }^{8}$ Leiden Observatory, Leiden University, PO Box 9513, 2300 RA Leiden, The Netherlands \\ ${ }_{9}^{9}$ Instituto de Astrofísica, Ponticia Universidad Católica de Chile, Av. Vicuña Mackenna 4860, 7820436 Macul, Santiago, Chile \\ ${ }^{10}$ Anton Pannekoek Institute for Astronomy, University of Amsterdam, Science Park 904, 1098 XH, Amsterdam, The Netherlands \\ ${ }^{11}$ Institute of Astronomy, University of Cambridge, Madingley Road, Cambridge CB3 OHA, UK \\ ${ }^{12}$ Laboratory for Astrophysics, Leiden Observatory, Leiden University, PO Box 9513, 2300 RA Leiden, The Netherlands \\ ${ }^{13}$ School of Physics and Astronomy, University of Leeds, Leeds LS2 9JT, UK \\ Received 2020 July 31; revised 2020 October 11; accepted 2020 November 2; published 2020 December 22
}

\begin{abstract}
Molecular D/H ratios are frequently used to probe the chemical past of solar system volatiles. Yet it is unclear which parts of the solar nebula hosted an active deuterium fractionation chemistry. To address this question, we present 0!"2-0!" 4 Atacama Large Millimeter/submillimeter Array (ALMA) observations of DCO ${ }^{+}$and DCN 2-1, 3-2, and 4-3 toward the nearby protoplanetary disk around TW Hya, taken as part of the TW Hya Rosetta Stone project, augmented with archival data. $\mathrm{DCO}^{+}$is characterized by an excitation temperature of $\sim 40 \mathrm{~K}$ across the 70 au radius pebble disk, indicative of emission from a warm, elevated molecular layer. Tentatively, DCN is present at even higher temperatures. Both $\mathrm{DCO}^{+}$and $\mathrm{DCN}$ present substantial emission cavities in the inner disk, while in the outer disk the $\mathrm{DCO}^{+}$and $\mathrm{DCN}$ morphologies diverge: most DCN emission originates from a narrow ring peaking around $30 \mathrm{au}$, with some additional diffuse $\mathrm{DCN}$ emission present at larger radii, while $\mathrm{DCO}^{+}$is present in a broad structured ring that extends past the pebble disk. Based on a set of simple parametric disk abundance models, these emission patterns can be explained by a near-constant DCN abundance exterior to the cavity, and an increasing $\mathrm{DCO}^{+}$abundance with radius. In conclusion, the ALMA observations reveal an active deuterium fractionation chemistry in multiple disk regions around TW Hya, but not in the cold planetesimalforming midplane and in the inner disk. More observations are needed to explore whether deuterium fractionation is actually absent in these latter regions, and if its absence is a common feature or something peculiar to the old TW Hya disk.
\end{abstract}

Unified Astronomy Thesaurus concepts: Planet formation (1241); Protoplanetary disks (1300); Millimeter astronomy (1061); Astrochemistry (75); Submillimeter astronomy (1647); Interstellar molecules (849); Solar system formation (1530)

\section{Introduction}

Solar system volatiles and organics are often observed to have nonsolar isotopic compositions, especially with respect to their D/H ratios (Robert \& Epstein 1982; Mumma \& Charnley 2011; Ceccarelli et al. 2014; Alexander et al. 2017; Altwegg et al. 2019). The observed deuterium enrichments encode information about the molecules' formation environment. They can therefore be used to differentiate between inheritance of material from the presolar molecular cloud, and in situ formation in the solar nebula (e.g., Cleeves et al. 2014). Differences in $\mathrm{D} / \mathrm{H}$ ratios between different solar system reservoirs have also been used to constrain the origins of Earth's water (Hartogh et al. 2011; Altwegg et al. 2015), and to infer that solar nebula chemistry, especially in the inner solar nebula, resulted in a reduction of $\mathrm{D} / \mathrm{H}$ levels in inherited, initially deuterium-rich volatiles (Yang et al. 2013; Furuya et al. 2017). Despite decades of solar system measurements and models, our understanding of deuterium fractionation chemistry and the distribution of deuterated volatiles in the solar nebula are, however, limited. Observations of deuterated species in analogs to the solar nebula, i.e., in protoplanetary disks, are key to anchor our models of deuterium fractionation chemistry in disks, and to put solar system measurements in context.

To date, four deuterated isotopologues have been detected in protoplanetary disks at millimeter wavelengths, $\mathrm{DCO}^{+}, \mathrm{DCN}$, $\mathrm{N}_{2} \mathrm{D}^{+}$, and $\mathrm{C}_{2} \mathrm{D}$ (Dutrey et al. 1996; van Dishoeck et al. 2003; Thi et al. 2004; Guilloteau et al. 2006; Qi et al. 2008; Fuente et al. 2010; Öberg et al. 2010, 2011, 2012; Mathews et al. 2013; Teague et al. 2015; Huang \& Öberg 2015; Qi et al. 2015; Öberg et al. 2015; Huang et al. 2017; Salinas et al. 2017; Loomis et al. 2020). When observed, the distributions of deuterated and nondeuterated isotopologues are frequently different, which implies some in situ formation (Huang et al. 2017). In other words, these observations show that there is an active deuterium fractionation chemistry in at 
Table 1

Observational Details of $\mathrm{DCO}^{+}$and DCN Lines

\begin{tabular}{|c|c|c|c|c|c|c|c|c|}
\hline Line & Date & $\begin{array}{l}\text { Int. Time } \\
\text { (min) }\end{array}$ & \# Ant. & $\begin{array}{l}\text { Baselines } \\
(\mathrm{m})\end{array}$ & $\begin{array}{l}\text { Ang. Res. } \\
\left({ }^{\prime \prime}\right)\end{array}$ & $\begin{array}{l}\text { Max. Ang. Scale } \\
\left({ }^{\prime \prime}\right)\end{array}$ & Phase Cal. & Flux Cal. \\
\hline \multirow[t]{3}{*}{$\mathbf{J}=2-1$} & 2016 Oct 22 & 46 & 48 & $19-1396$ & 0.37 & 3.8 & J1037-2934 & J1037-2934 \\
\hline & 2016 Oct 25 & 46 & 48 & 19-1396 & 0.37 & 3.8 & J1037-2934 & J1107-4449 \\
\hline & 2016 Oct 27 & 46 & 48 & 19-1396 & 0.37 & 3.8 & J1037-2934 & J1107-4449 \\
\hline \multirow[t]{3}{*}{$\mathrm{J}=3-2$} & 2016 Dec 16 & 24 & 45 & $15-460$ & 0.71 & 6.4 & J1037-2934 & J1107-4449 \\
\hline & 2017 May 5 & 40 & 45 & $15-1124$ & 0.29 & 4.2 & J1037-2934 & J1037-2934 \\
\hline & 2017 May 7 & 40 & 50 & $15-1124$ & 0.28 & 3.9 & J1037-2934 & J1037-2934 \\
\hline \multirow[t]{3}{*}{$1 \mathrm{~J}=4-3$} & 2017 Feb 1 & 29 & 41 & $15-260$ & 0.89 & 7.6 & J1037-2934 & J1107-4449 \\
\hline & 2018 Jan 23 & 48 & 43 & $15-1398$ & 0.20 & 2.9 & J1037-2934 & J1037-2934 \\
\hline & 2018 Sep 20 & 48 & 44 & $15-1398$ & 0.20 & 3.0 & J1037-2934 & J0904-5735 \\
\hline
\end{tabular}

least some disk locations during planet formation. Whether this chemistry impacts the deuterium levels in volatiles in the disk midplane where planetesimals assemble depends on which disk layer is producing the observed deuterated isotopologue emission and the efficiency of vertical mixing in disks.

A priori $\mathrm{DCO}^{+}$and $\mathrm{DCN}$ emission may originate from a range of disk layers because there are multiple potential formation pathways for each molecule (e.g., Millar et al. 1991; Aikawa \& Herbst 1999; Turner 2001; Willacy 2007; Favre et al. 2015; Aikawa et al. 2018; Roueff et al. 2015). In general, pathways that begin with the formation of $\mathrm{H}_{2} \mathrm{D}^{+}$are active at low temperatures, $<30 \mathrm{~K}$, characteristic of regions close to the disk midplane, while pathways that begin with deuterated small hydrocarbons, initiated by the $\mathrm{CH}_{2} \mathrm{D}^{+}$ion, can operate at a larger range of temperatures (Wootten 1987; Parise et al. 2009; Roueff et al. 2015), including in inner disk regions and in disk atmospheres. Most spatially resolved observations of $\mathrm{DCO}^{+}$ show extended emission in the outer disk, and a lack of emission in the inner disk (Qi et al. 2008; Öberg et al. 2015; Huang et al. 2017; Salinas et al. 2017), which is most consistent with formation through the colder $\mathrm{H}_{2} \mathrm{D}^{+}$pathway. This conclusion was supported by a measurement of the $\mathrm{DCO}^{+}$ excitation temperature in the disk around HD 163296, which was estimated to 12-20 K (Flaherty et al. 2017). However, contributions from the $\mathrm{CH}_{2} \mathrm{D}^{+}$pathway cannot generally be excluded, and Carney et al. (2018) found that in the case of the disk around HD 169142, the majority of observed $\mathrm{DCO}^{+}$ emission originates from a warmer disk region. DCN emission generally, but not always, appears radially more compact than $\mathrm{DCO}^{+}$(Öberg et al. 2012; Huang et al. 2017; Salinas et al. 2017), indicative of a larger contribution from the warmer formation pathways. There are no direct measurements of the DCN excitation temperature in a disk.

In this paper, we use Atacama Large Millimeter/ submillimeter Array (ALMA) observations of multiple $\mathrm{DCO}^{+}$ and DCN lines to map out the $\mathrm{DCO}^{+}$and DCN distributions and excitation temperatures across the nearby protoplanetary disk around TW Hya. TW Hya is an excellent benchmarking system because of extensive structural and chemical modeling (e.g., Bergin et al. 2013; Cleeves et al. 2015). The present study uses data from the TW Hya Rosetta Stone Project along with other archival date sets. The Rosetta program set out to map chemistry at 10 au resolution to understand the spatial distribution of commonly observed molecules and their isotopologues toward TW Hya. The project as a whole will inform studies at lower resolution, which in some cases is unavoidable for more distant protoplanetary disks. Section 2 summarizes the observational details and the data reduction procedure. Section 3 presents the observational data products, disk-averaged and radially resolved column densities, and excitation temperatures using rotational diagrams. Informed by the rotational diagram analysis, Section 4 introduces a series of toy models, generated using RADMC-3D (Dullemond 2012), aimed at qualitatively exploring what kind of 2D abundance structures can explain the observations. We discuss the observations and modeling results in Section 5, followed by a summary and some concluding remarks in Section 6.

\section{Observational Details}

$\mathrm{DCO}^{+}$and DCN J = 3-2 and 4-3 observations toward TW Hya were acquired as a part of the Rosetta Stone project (2016.1.00311.S and 2017.1.00769.S; PI: L. I. Cleeves) in six separate executions. The two $\mathrm{J}=3-2$ lines were observed together in one science goal, and the two $\mathrm{J}=4-3$ lines in a second one. Observations of $\mathrm{DCO}^{+}$and $\mathrm{DCN} 2-1$ were obtained as part of project 2016.1.00440.S (PI: R. Teague) in three separate executions. The observation dates, integration times, number of antennas, range of baselines, nominal angular resolutions, maximum recoverable angular scales, phase calibrators, and flux calibrators are listed in Table 1

The measurement sets associated with each execution were initially calibrated by the ALMA staff. Additional selfcalibration was applied to the pipeline calibrated data for the TW Hya Rosetta Stone observations using CASA 4.5. Phaseonly self-calibration was conducted on the line-free continuum using $30 \mathrm{~s}$ integrations and averaging both polarizations. The solutions were applied to the full-resolution measurement sets. The line data were continuum subtracted using uvcontsub and imaged with the CLEAN algorithm (Högbom 1974). The 3-2 and 4-3 line observations were cleaned with a $0.07 \mathrm{~km} \mathrm{~s}^{-1}$ velocity resolution and the $2-1$ with a $0.35 \mathrm{~km} \mathrm{~s}^{-1}$ resolution down to a level of $2 \times$ rms. During the CLEAN process, we employed a mask, constructed by manually identifying areas with emission in each channel, and a Briggs parameter of 0.5 for the fiducial image cubes. The resulting beam sizes, peak line emission, and $\mathrm{rms}$ in $\mathrm{Jy} \mathrm{beam}^{-1}$ are presented in Table 2.

We also produced a second set of image cubes with the resolution of all lines smoothed using imsmooth in the Common Astronomy Software Applications package (CASA) with a circular 0.15 beam. This resolution matches the major axis of the beams in the 3-2 line data and thus constitute the highest uniform resolution we can achieve across the sample with a circular beam. These image cubes are used in the quantitative line analysis in Section 3.2 where a uniform resolution for the $2-1,3-2$, and 4-3 lines is required. 

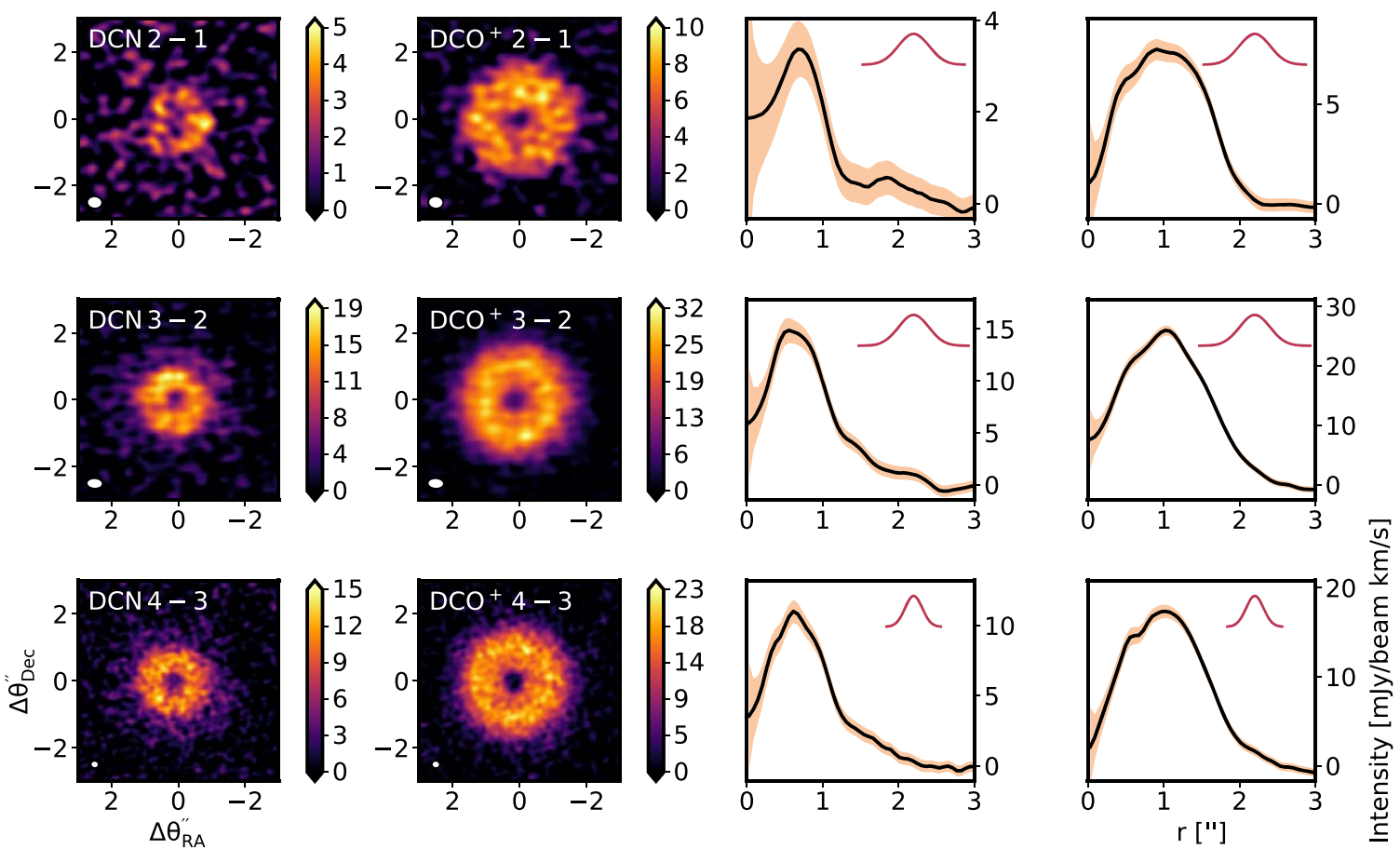

Figure 1. Left two panels: integrated emission maps of $\mathrm{DCN}$ and $\mathrm{DCO}^{+} 2-1,3-2$, and 4-3 lines toward TW Hya. Beam sizes are in the bottom left corner of each panel. Right two panels: azimuthally averaged radial intensity profiles of the same lines, with restored beam sizes plotted in each panel. Note the narrow ring and the diffuse halo for $\mathrm{DCN}$, and inner plateu and the extended ring structure of $\mathrm{DCO}^{+}$. The translucent color shows the $1 \sigma$ confidence intervals, not accounting for absolute flux uncertainties.

Table 2

Line Catalog and Observational Data

\begin{tabular}{|c|c|c|c|c|c|c|c|c|c|}
\hline Line & $\begin{array}{l}\text { Rest Freq. } \\
(\mathrm{GHz})\end{array}$ & $\log _{10}\left(A_{i j}\right)$ & $\begin{array}{c}E_{\mathrm{u}} \\
(\mathrm{K})\end{array}$ & $g_{\mathrm{u}}$ & $\begin{array}{l}\text { Beam (PA) } \\
\left(" \times{ }^{\prime \prime}\left({ }^{\circ}\right)\right)\end{array}$ & $\begin{array}{c}\mathrm{rms}^{\mathrm{b}} \\
\left(\mathrm{mJy} \mathrm{beam}^{-1}\right)\end{array}$ & $\begin{array}{c}\text { Flux }^{\mathrm{c}} \\
\left(\mathrm{mJy} \mathrm{km} \mathrm{s}^{-1}\right)\end{array}$ & $\begin{array}{l}\text { Flux }<100 \text { au } \\
\left(\mathrm{mJy} \mathrm{km} \mathrm{s}^{-1}\right)\end{array}$ & $\begin{array}{c}\Delta v^{\mathrm{f}} \\
\left(\mathrm{km} \mathrm{s}^{-1}\right)\end{array}$ \\
\hline $\mathrm{DCO}^{+} \mathrm{J}=2-1$ & $144.077285^{\mathrm{d}}$ & -3.67 & 10.37 & 5 & $0 ! .42 \times 00^{\prime \prime} 34\left(84^{\circ}\right)$ & 1.2 & $559 \pm 16$ & $471 \pm 11$ & $2.1-3.5$ \\
\hline $\mathrm{DCO}^{+} \mathrm{J}=3-2$ & $216.112582^{\mathrm{d}}$ & -3.12 & 20.74 & 7 & $0 . " 49 \times 0 . .31\left(88^{\circ}\right)$ & 3.4 & $1904 \pm 19$ & $1532 \pm 13$ & $2.1-3.5$ \\
\hline $\mathrm{DCN} J=2-1$ & $144.827996^{\mathrm{d}}$ & -3.89 & 10.42 & 15 & $0 ! .42 \times 0 . " 34\left(85^{\circ}\right)$ & 1.1 & $>128 \pm 12^{\mathrm{e}}$ & $>101 \pm 8^{\mathrm{e}}$ & $2.1-3.5$ \\
\hline $\mathrm{DCN} \mathrm{J}=3-2$ & $217.238537^{\mathrm{d}}$ & -3.34 & 20.85 & 21 & $0 ! \prime 48 \times 0 . ! 31\left(88^{\circ}\right)$ & 3.3 & $641 \pm 20$ & $561 \pm 13$ & $2.2-4.0$ \\
\hline $\mathrm{DCN} \mathrm{J}=4-3$ & $289.644917^{\mathrm{d}}$ & -2.95 & 34.75 & 27 & $0 . .24 \times 0 . .22\left(80^{\circ}\right)$ & 2.3 & $1344 \pm 29$ & $1206 \pm 19$ & $2.0-3.7$ \\
\hline
\end{tabular}

Note.

${ }^{\mathrm{a}}$ Line catalog data from CDMS (Müller et al. 2005)

${ }^{\mathrm{b}}$ In $0.07 \mathrm{~km} \mathrm{~s}^{-1}$ channels for 3-2 and 4-3 lines, and in $0.35 \mathrm{~km} \mathrm{~s}^{-1}$ channels for 2-1 lines

${ }^{c}$ Integrated out to $2{ }^{\prime \prime} 5$. Uncertainty does not include the $10 \%$ flux calibration uncertainty.

${ }^{\mathrm{d}}$ There is no visible $\mathrm{DCO}^{+}$and DCN fine structure in our data.

e The DCN 2-1 line emission is likely underestimated due to low $\mathrm{S} / \mathrm{N}$ and some of the emission being carried by fine structure lines, which are not included.

${ }^{\mathrm{f}}$ The velocity range over which emission is integrated.

\section{Results}

\subsection{DCN and DCO Emission Maps and Spectra}

Figure 1 shows integrated emission (moment 0 ) maps and radial profiles of the $\mathrm{DCN}$ and $\mathrm{DCO}^{+} \mathrm{J}=2-1,3-2$, and 4-3 rotational line emission toward TW Hya. The maps are constructed by integrating emission across all channels that show signal above $3 \sigma$ (Table 2). We do not detect any molecular line hyperfine structure, i.e., it is either too weak or unresolved. The radial profiles are derived from the momentzero maps by azimuthally integrating the inclination-corrected maps $\left(i=7^{\circ}\right.$ and P.A. $\left.=155^{\circ}\right)$ in narrow rings. These values are slightly different from those used by Huang et al. (2018), and were selected because they provided the best visual fit to the data when overplotting a Keplerian model on top of the channel maps (see the Appendix). Displayed uncertainties are extracted using the rms per beam in the maps and taking into account the number of independent beams in each ring.

All $\mathrm{DCO}^{+}$and DCN moment-zero maps show qualitatively similar central depressions or holes in the line emission. The central holes are not identical for $\mathrm{DCO}^{+}$and $\mathrm{DCN}$, however. Figure 2 shows an overlay of the higher resolution 4-3 lines, which indicate that the DCN hole has a somewhat smaller radius. On closer inspection, it seems like DCN and $\mathrm{DCO}^{+}$ share a radial component that peaks at $\sim 00^{\prime \prime} 6$. Interior to this, DCN has a shoulder at $\sim 0$ ". 4 . Exterior to 0". 6 the emission pattern clearly differs for $\mathrm{DCN}$ and $\mathrm{DCO}^{+}$. The $\mathrm{DCN}$ emission decreases quickly with radius, followed by a low-intensity halo stretching out to larger radii. $\mathrm{DCO}^{+}$displays a broad second component that encompasses the DCN halo. This second 


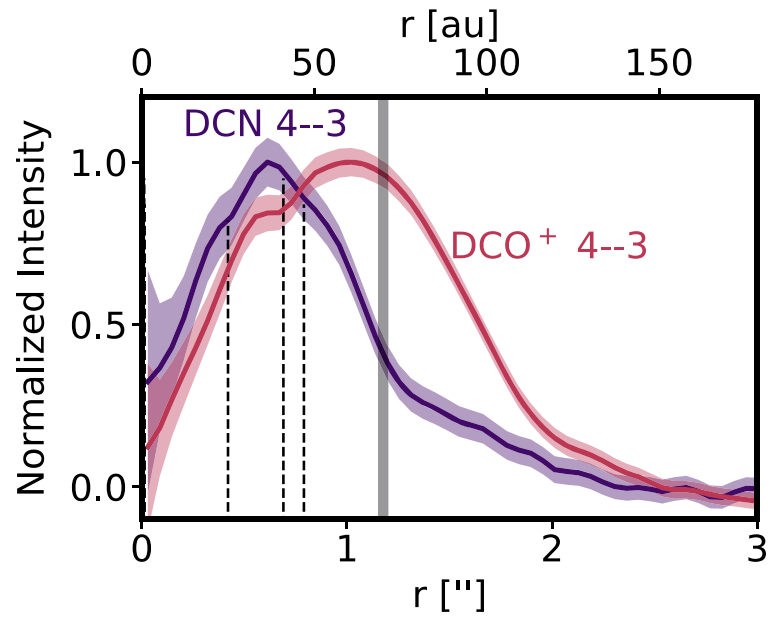

Figure 2. DCN and $\mathrm{DCO}^{+} 4-3$ radial profiles normalized to the maximum flux. Note the different inner disk hole sizes. The dashed lines mark previously observed dust gaps at 25,41 , and 47 au or $0 . / 42,0$. . 69, and 0.79 (Huang et al. 2018) and the broad, translucent line marks the edge of the pebble disk.

$\mathrm{DCO}^{+}$component peaks at $\sim 1^{\prime \prime}$. None of these features correspond to previously noted dust rings, but we note that the DCN shoulder at $\sim 0$ ". 4 or $\sim 25$ au coincides with a dust gap, and a break in the ${ }^{12} \mathrm{CO}$ radial emission profile (Huang et al. 2018). Furthermore, the DCN peak nearly coincides with a second dust gap at 41 au $(\sim 0$ !" 69$)$. The precise dust and gas properties of these gaps are unknown, and it is therefore unclear if the dust gaps are causing an increased DCN emission. One possible causal connection is that dust gaps likely present increased UV penetration, and therefore increased dissociation of $\mathrm{CO}$ in carbon atoms, which could fuel nitrile production. If this is the case, we would expect future observations to reveal excess emission of other nitriles at the same locations.

The emission morphologies of each molecule are consistent across the different transitions. This consistency indicates that all lines of each molecule likely originate in the same disk regions, and that observed emission substructures trace changes in column density, and not only changes in excitation conditions across the disk. We cannot rule out that some of the missing $\mathrm{DCO}^{+}$and $\mathrm{DCN}$ emission toward the disk center is due to continuum opacity, since Huang et al. (2018) finds that the continuum $<20$ au may be, in part, optically thick. Continuum opacity cannot be the whole explanation for these central cavities, however, since other optically thin lines, including ${ }^{13} \mathrm{C}^{18} \mathrm{O}$, present centrally peaked emission (Zhang et al. 2017) this suggests a chemical cause for the central cavities, for example the turn-off of the dominant deuterium fractionation pathway; this is discussed in Section 5.

Table 2 lists the integrated emission across the entire $\mathrm{DCO}^{+}$ disk (out to 2!"5) and within a radius of 1 !"7 or 100 au (where most $\mathrm{DCO}^{+}$and DCN emission originates). The listed uncertainties are based on the measured rms per beam in line emission-free moment-zero maps, produced from line-free channels in the relevant spectral window, multiplied by the square root of the number of beams within $r=2 ! .5$ and 1 !'7, respectively. We suspect that the DCN 2-1 line emission is underestimated due to a combination of unaccounted emission from hyperfine line emission and incomplete flux recovery of this low signal-to-noise ratio $(\mathrm{S} / \mathrm{N})$ line.
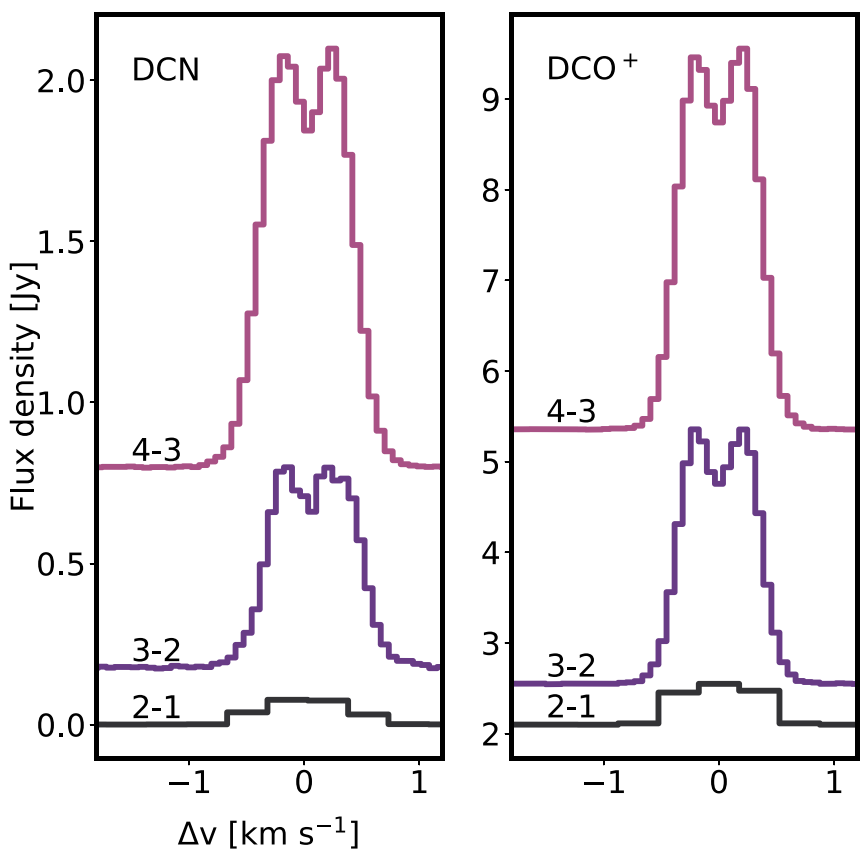

Figure 3. $\mathrm{DCN}$ and $\mathrm{DCO}^{+}$spectra extracted using Keplerian masks. The spectra are offset for clarity.

Figure 3 shows the extracted spectra of the DCN and $\mathrm{DCO}^{+}$ lines using Keplerian masks (Pegues et al. 2020) to enhance the $\mathrm{S} / \mathrm{N}$. The 4-3 and 3-2 lines show Keplerian profiles, while the resolution of the 2-1 lines are too poor to resolve the characteristic double-peak structure. The DCN lines are broader than the $\mathrm{DCO}^{+}$lines, which has two origins: an unresolved hyperfine structure and more emission emitting at smaller disk radii. We also inspected spectra from individual pixels and saw no evidence for substantial non-Keplerian motion or line self-absorption.

\subsection{Rotational Diagram Analysis of $\mathrm{DCO}^{+}$}

We begin our characterization of $\mathrm{DCO}^{+}$and DCN using diskaveraged rotational diagrams. We use the $\mathrm{DCN}$ and $\mathrm{DCO}^{+}$ integrated fluxes within $100 \mathrm{au}$, which encompass the main emission features. We exclude the DCN 2-1 emission from the rotational diagram analysis because it is likely underestimated (see the discussion above)—if it is included, the derived temperature is above $100 \mathrm{~K}$ and the fit to the other lines is poor. The main flux uncertainty is a $10 \%$ absolute flux calibration uncertainty, which is added in quadrature to the rms-based integrated emission errors when calculating the rotational diagram. The molecular line data was all taken from the Cologne Database for Molecular Spectroscopy (CDMS) and is listed in Table 2. We used the following partition functions (also from CDMS) calculated at $[0,9.375,18.75,37.5,75,150$, 225, 300] K: [0, 5.769, 11.1866, 22.0293, 43.7220, 87.1365, $130.5570,173.9803]$ and $[0,17.2240,33.3906,65.7550$, 130.5095, 262.2213, 409.8604, 586.3727] for $\mathrm{DCO}^{+}$and DCN, respectively.

To calculate the rotational diagrams, we follow the Markov Chain Monte Carlo (MCMC) procedure outlined in Loomis et al. (2018a), using the emcee package (Foreman-Mackey et al. 2013). Figure 4 shows rotational diagrams corresponding to random draws from the posterior probability distributions of the excitation temperatures and column densities, with the 

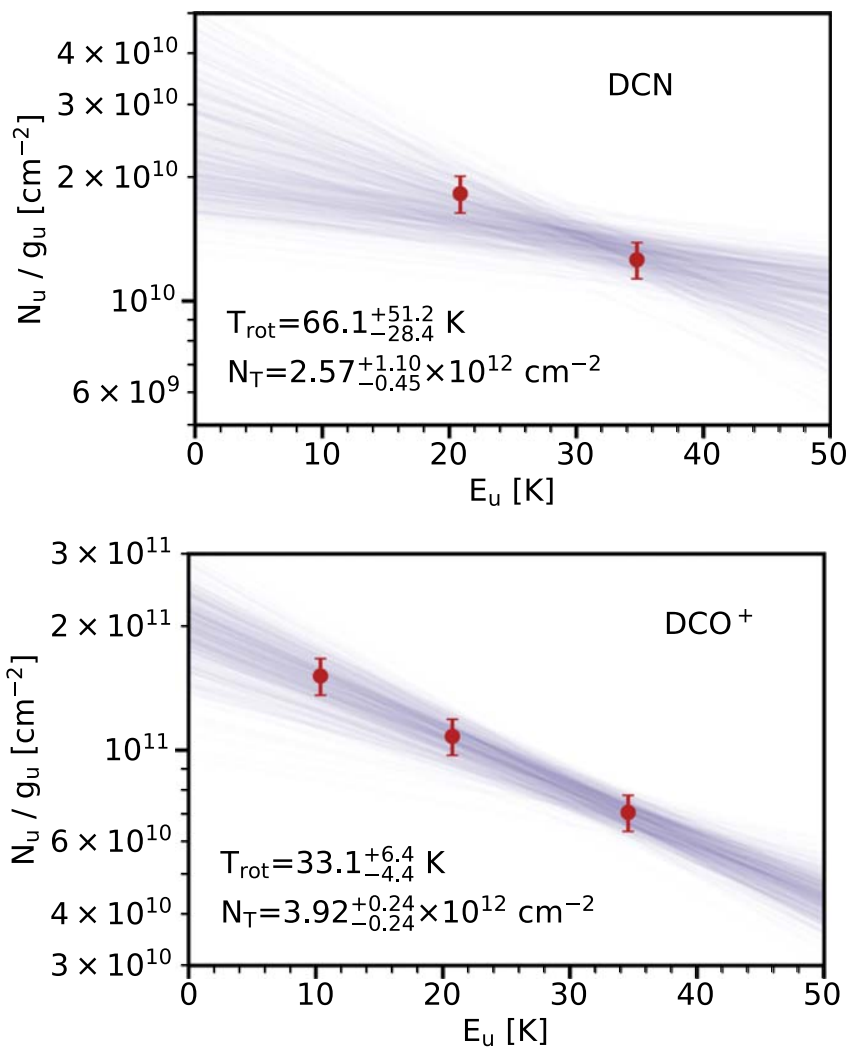

Figure 4. Rotational diagram of disk-averaged $\mathrm{DCN}$ and $\mathrm{DCO}^{+}$lines toward TW Hya out to a disk radius of 100 au. Note that the DCN rotational diagram is uncertain because it is based on only the 3-2 and 4-3 lines.

optical depth corrected values of $N_{\mathrm{u}} / g_{\mathrm{u}}$ plotted against $E_{\mathrm{u}}$. The disk-averaged column densities of both molecules are similar: 2.6 and $3.9 \times 10^{12} \mathrm{~cm}^{-2}$, respectively for $\mathrm{DCN}$ and $\mathrm{DCO}^{+}$. $\mathrm{DCO}^{+}$appears colder than DCN (33 versus $66 \mathrm{~K}$ ), but we note that the fit to the DCN data is uncertain since we had to remove the 2-1 line, and we cannot exclude that the actual DCN excitation temperature is $<40 \mathrm{~K}$.

We next use $\mathrm{DCO}^{+} 4-3,3-2$, and 2-1 radial emission profiles (Figure 5, top panel) to carry out the same rotational diagram procedure in radial bins across the disk. The radial profiles were generated from moment-zero maps with a resolution of 0.15 , and hence appear smoother than the profiles in Figure 1. Similar to the disk-averaged rotational analysis, the absolute calibration uncertainty was added in quadrature to the rms-based uncertainty shown in Figure 5. The middle and bottom panels of Figure 5 show the resulting radial temperature and column density profiles. $\mathrm{DCO}^{+}$appears to display a rapid decrease in excitation temperature within the inner gap, i.e., out to $\sim 25 \mathrm{au}$, but the emission levels within the gap are low and the uncertainties are too high to claim a certain trend. Between 25-70 au, the best-fit $\mathrm{DCO}^{+}$excitation temperatures are $37-39.5 \mathrm{~K}$, suggesting that in the bulk of the disk, $\mathrm{DCO}^{+}$ originates in a layer that is more elevated than the $\mathrm{CO}$ snow surface, which is expected at $\sim 20-25 \mathrm{~K}$. Beyond 70 au, which coincides with the outer edge of the pebble disk (Andrews et al. 2012, 2016; Huang et al. 2018), the $\mathrm{DCO}^{+}$temperature begins to drop and reaches $<30 \mathrm{~K}$ at $90 \mathrm{au}$. At even larger radii there is a possible temperature inversion, but the $\mathrm{S} / \mathrm{N}$ is too low to determine that this is real. We note that the lower $\mathrm{DCO}^{+}$ excitation temperature in the outer disk has a disproportional impact on the disk-averaged excitation temperature due to the relatively larger emission area of the 70-120 au portion of the disk compared to the inner $70 \mathrm{au}$, which explains why the disk-averaged $\mathrm{DCO}^{+}$excitation temperature is lower than the characteristic excitation temperature.

The rotational diagram results have two important caveats. First the emission surfaces of the different $\mathrm{DCO}^{+}$transitions are not necessarily the same, and this may skew the temperature upwards. We explore below whether the $\mathrm{DCO}^{+}$emission could be consistent with a colder origin. Second, the rotational diagram analysis as well as the parametric models below, assume local thermal equilibrium (LTE), and if some of the $\mathrm{DCO}^{+}$is originating from very elevated disk layers this assumption may not hold. While we cannot exclude non-LTE excitation, we note that it typically results in subthermally excited lines and therefore an underprediction of the kinetic temperature, and should therefore not impact the main result here that $\mathrm{DCO}^{+}$appears to originate from a warmer disk layer than expected.

\section{Exploratory Parametric Models}

To explore what radial and vertical $\mathrm{DCO}^{+}$and $\mathrm{DCN}$ distributions can qualitatively explain the observations presented above, we construct a series of toy models with parametric $\mathrm{DCN}$ and $\mathrm{DCO}^{+}$abundance profiles. We first construct a simple disk density model using the common power-law prescription for the gas surface density and calculate the density using a radially dependent scale height to simulate disk flaring:

$$
\begin{aligned}
\Sigma_{\text {gas }} & =\Sigma_{\text {gas }, R=20 a u} \times(R / 20 \mathrm{au})^{\gamma}, \\
\rho & =\frac{\Sigma}{\sqrt{2 \pi} H} \exp \left(-\frac{1}{2}\left(\frac{z}{H}\right)^{2}\right) \\
H & =H_{R=20 \mathrm{au}} \times(R / 20 \mathrm{au})^{p} .
\end{aligned}
$$

The surface density normalization $\Sigma_{\text {gas }, R=20 a u}$ and power-law index $\gamma$ are set to $35 \mathrm{~g} \mathrm{~cm}^{-2}$ and -1.3 , respectively, to mimic the surface density model presented in Cleeves et al. (2015). Following Cleeves et al. (2015), we set the scale height normalization factor as $H_{R=20 \mathrm{au}}=2$ au and the flaring index as $p=0.3$. We adopt a simple power law for the disk midplane temperature, using the normalization factor and power-law index derived in Zhang et al. (2017). To convert from density to number density, we adopted a mean molecular weight of 2.37, which takes into account that the hydrogen is mainly molecular. In elevated disk layers we parameterize the gas temperature using the common prescription from Dartois et al. (2003), which takes into account direct gas heating in the lower density upper disk layers,

$$
\begin{gathered}
T_{\text {mid }}=40 \times(R / 10 \mathrm{au})^{-0.47}, \\
T_{\text {atm }}=125 \times(R / 10 \mathrm{au})^{-0.47}, \\
T_{R, z}=T_{\text {atm }}+\left(T_{\text {mid }}-T_{\text {atm }}\right) \times \cos (\pi z /(8 H))^{4} .
\end{gathered}
$$

The normalization temperature of the atmosphere of $125 \mathrm{~K}$ follows Huang et al. (2018) and is in reasonable agreement with the atmospheric temperature used by Cleeves et al. (2015). The resulting gas density and temperature structures are shown in Figure 6.

Using this simple parametric disk structure model, we evaluate different parametric $\mathrm{DCO}^{+}$and $\mathrm{DCN}$ abundance models that are based on a combination of power-law prescriptions, radial cutoffs, and temperature boundaries as tabulated in Table 3. In each case we simulate noiseless ALMA observations for the 2-1 and 

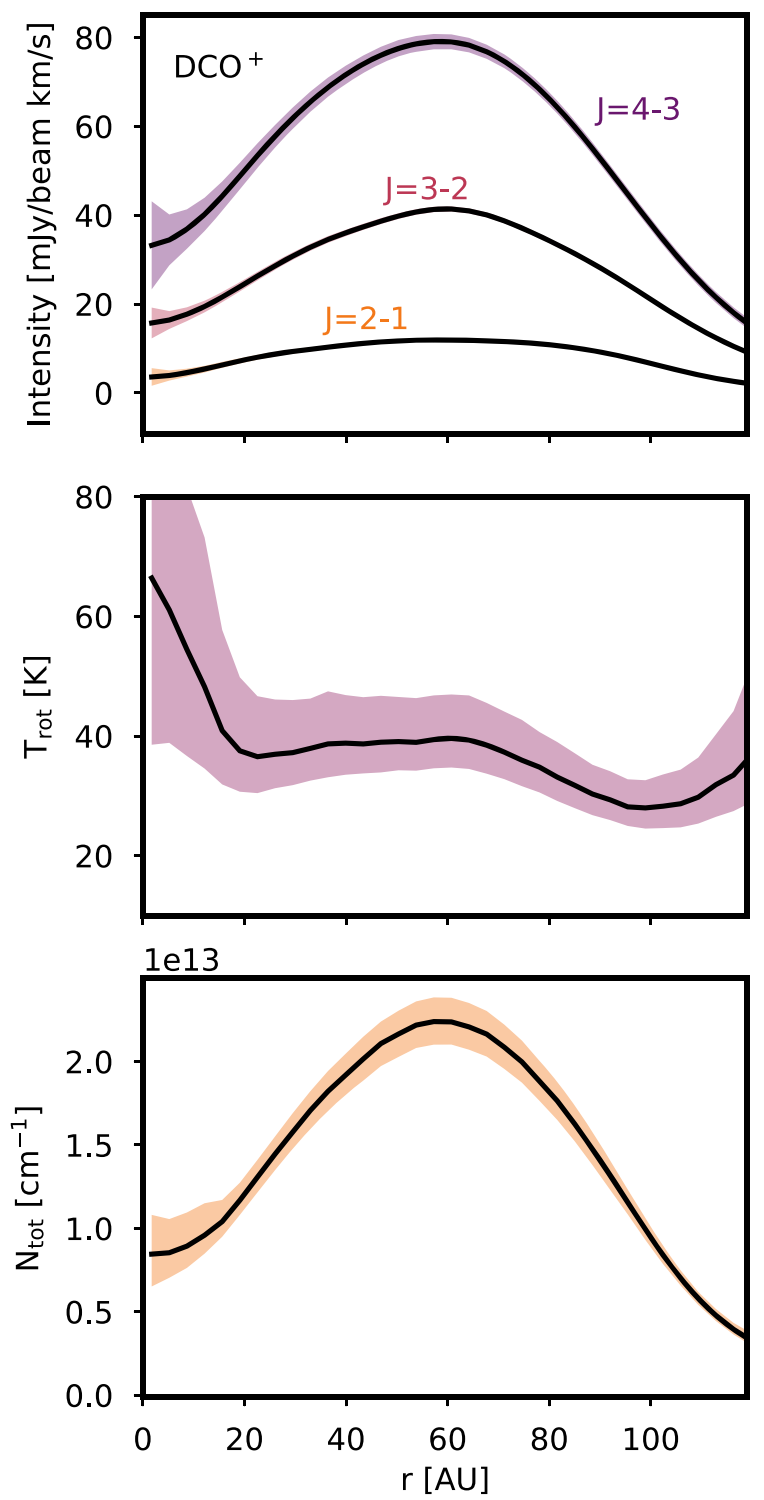

Figure 5. Radially resolved rotational diagram analysis for $\mathrm{DCO}^{+}$. Top: radial emission profiles at a resolution of $0 . .5$ of the $\mathrm{DCO}^{+}$lines. Middle: derived excitation temperatures with confidence intervals corresponding to the 16th to 84th percentiles. Bottom: calculated column density profile with confidence intervals.

4-3 lines using RADMC-3D (Dullemond 2012) for the radiative transfer, vis_sample for the visibility sampling (Loomis et al. 2018b), and then tclean in CASA. We used the $\mathrm{DCO}^{+}$ molecular file from the Leiden Atomic and Molecular Database (LAMDA; Schöier et al. 2005) and created our own DCN molecular file using frequencies and energy level data from CDMS (Müller et al. 2005). We also included the 3-2 lines in initial model runs, but found that they did not add much to this qualitative model-data comparison due to the small difference in energy levels between the 4-3 and 3-2 lines. The simulated observations are analyzed using the same procedure as applied to our ALMA observations to enable direct comparison between observed and simulated line emission radial profiles.

\subsection{Radial Boundary Models}

The first set of models focuses on the inner cavity seen in both $\mathrm{DCO}^{+}$and $\mathrm{DCN}$ emission. The first abundance model

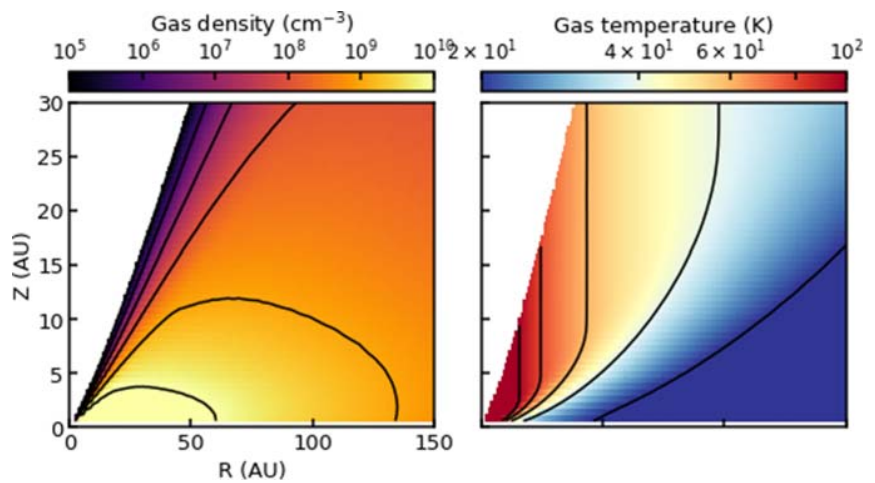

Figure 6. Parametric number density and temperature distributions used to qualitatively evaluate different $\mathrm{DCO}^{+}$and DCN abundance models in the TW Hya disk.

(A1) has a constant abundance exterior to $25 \mathrm{au}$, and five orders of magnitude lower abundance in the inner hole. The abundance exterior to 25 au is estimated by eye to fit the 4-3 lines. Figures 7 (first panel) and 8 (first panel) show that this prescription does not provide a good fit to either $\mathrm{DCO}^{+}$or DCN emission, but for different reasons. In the case of $\mathrm{DCO}^{+}$, the inner part of the emission profile is well fit by Model A1, while the outer disk is not, indicative that the $\mathrm{DCO}^{+}$abundance is higher in the outer than in the inner disk. In the case of DCN, this model produces too little emission in the hole and too much emission at large radii, beyond 60 au.

We first address the $\mathrm{DCO}^{+}$discrepancy by exploring whether changing the abundance profile exterior to the hole from constant to an increasing power law provides a better fit (Model A2). The second panel of Figure 7 shows that a DCO $^{+}$ abundance power law with a power-law index of 0.75 does indeed provide a reasonable fit to the $\mathrm{DCO}^{+}$emission profile when imaged at this resolution. Note, however, that our higher resolution data shows a $\mathrm{DCO}^{+}$double peak, rather than a broad single ring, which could not be explained by such a continuous power law. We also note that the model that fits the 4-3 data predicts a 2-1 emission level that is close to the one observed. This shows that our observations do not rule out the presence of $\mathrm{DCO}^{+}$close to the disk midplane, as long as there is a substantial amount of $\mathrm{DCO}^{+}$in the warm, upper disk layers.

The DCN A1 discrepancy indicates that the DCN abundance is lower in the outer disk than the inner disk if DCN is emitting from all disk layers. One possible explanation is that DCN is only emitting from warmer gas, of which there is a limited amount in the outer disk. To test this, we apply a $20 \mathrm{~K}$ temperature boundary to the A1 model (Model A3), i.e., a constant abundance exterior to 25 au wherever the temperature is $>20 \mathrm{~K}$, and a five orders of magnitude lower abundance everywhere else. In our disk model, the midplane drops below $20 \mathrm{~K}$ at $44 \mathrm{au}$. The second panel of Figure 8 shows that this model fits the 4-3 data quite well in the outer disk, but naturally does not fix the underabundance noted toward the disk center, which could be addressed either by making the inner cavity $\sim 5$ au smaller or by implementing a smaller DCN depletion factor (not shown). The $2-1$ data is always overpredicted, indicative that the DCN is even warmer.

\subsection{Temperature Boundary Models}

In a second set of of models we apply temperature boundaries, rather than radial cutoffs, with the aim of exploring 

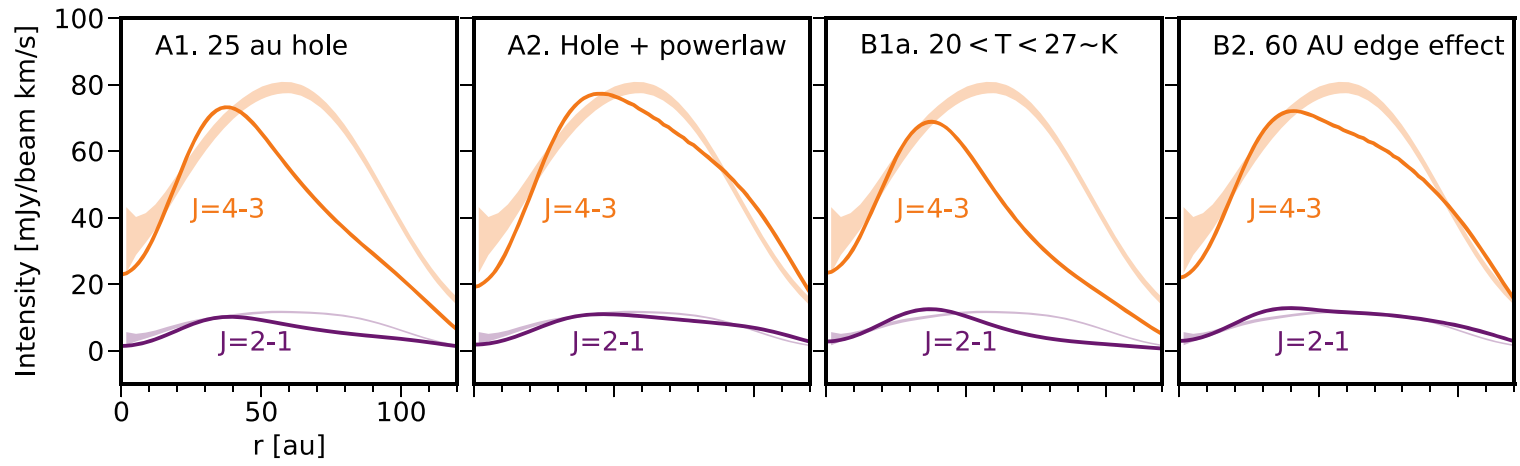

Figure 7. Comparison between observed (broad bands) and modeled (thin lines) radial emission profiles of the $\mathrm{DCO}^{+} 2-1$ and $4-3$ lines. The bandwidths of the observed profiles approximate the observational uncertainties, but do not include a $10 \%$ absolute calibration error.
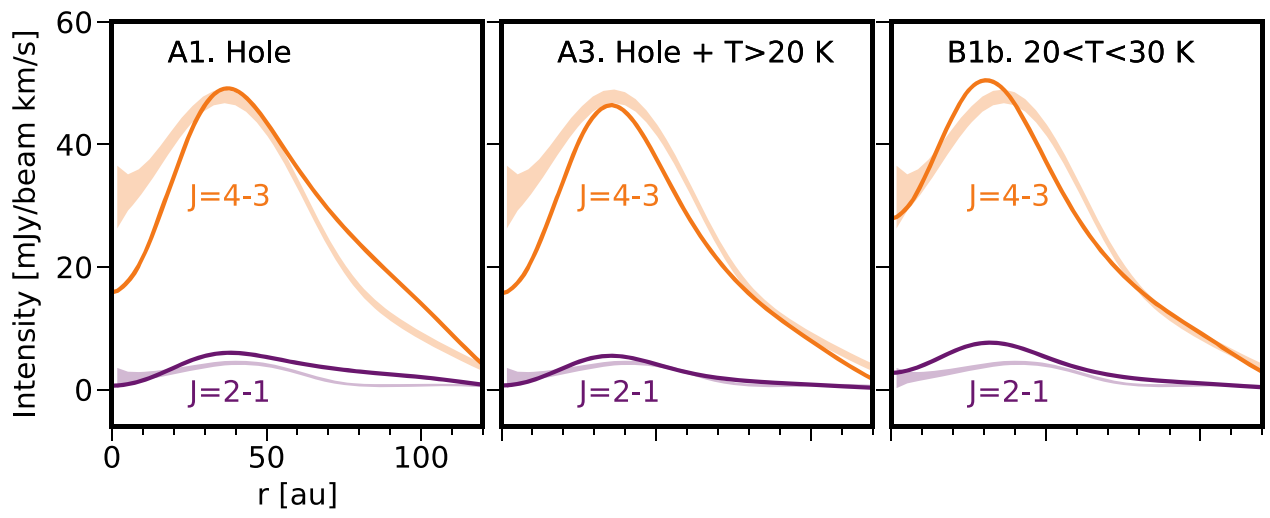

Figure 8. Comparison between observed (broad bands) and modeled (thin lines) radial emission profiles of the DCN 4-3 line. The bandwidths of the observed profiles approximate the observational uncertainties, but do not include a $10 \%$ absolute calibration error. Note that the observed DCN 2-1 line emission profile should be considered a lower limit for the reasons discussed in the text.

Table 3

Toy Model Parameters

\begin{tabular}{|c|c|c|c|c|c|c|}
\hline Model & Mol. & $n_{R=25 \mathrm{au}}\left(n_{\mathrm{H}}\right)$ & PL Index ${ }^{a}$ & $R_{\text {hole }}(\mathrm{au})$ & $T$ Boundaries $(\mathrm{K})$ & Special Constraints \\
\hline \multirow[t]{2}{*}{$\overline{\mathrm{A} 1}$} & $\mathrm{DCO}^{+}$ & $1.2 \times 10^{-12}$ & 0 & 25 & $\ldots$ & $\ldots$ \\
\hline & $\mathrm{DCN}$ & $1.2 \times 10^{-12}$ & 0 & 25 & $\ldots$ & $\ldots$ \\
\hline A2 & $\mathrm{DCO}^{+}$ & $8 \times 10^{-13}$ & 0.75 & 25 & $\ldots$ & $\ldots$ \\
\hline A3 & $\mathrm{DCN}$ & $1.2 \times 10^{-12}$ & 0 & 25 & $T>20$ & $\ldots$ \\
\hline B1a & $\mathrm{DCO}^{+}$ & $3.5 \times 10^{-12}$ & 0 & 0.1 & $20<T<27$ & $\ldots$ \\
\hline B1b & $\mathrm{DCN}$ & $2.4 \times 10^{-12}$ & 0 & 0.1 & $20<T<30$ & $\ldots$ \\
\hline B2 & $\mathrm{DCO}^{+}$ & $3.5 \times 10^{-12}$ & 0 & 0.1 & $20<T<27$ & at $R>60 \mathrm{au}, T<27 \mathrm{~K}$ \\
\hline
\end{tabular}

Note.

${ }^{\mathrm{a}}$ Power-law index for abundance profile

whether the observed emission profiles can be explained by DCN and $\mathrm{DCO}^{+}$temperature-dependent formation alone. The first temperature models (B1a and B1b) assume constant abundances within lower and upper temperature bounds across the disk. We explored several different boundaries, and found that the inner radial profile requires a maximum temperature cutoff at $25-30 \mathrm{~K} ; 27 \mathrm{~K}$ provides the best fit for $\mathrm{DCO}^{+}$(B1a), and $30 \mathrm{~K}$ for $\mathrm{DCN}(\mathrm{B} 1 \mathrm{~b})$. We set the temperature minimum cutoff of $20 \mathrm{~K}$, based on model predictions for $\mathrm{CO}$ freeze-out. The B1 model provides a good fit to the DCN 4-3 data, but overpredicts the $2-1$ line emission. The $2-1$ emission is also overpredicted for $\mathrm{DCO}^{+}$in the inner disk by $20-30 \%$. This is qualitatively consistent with the results from the rotational diagram analysis, which indicated that a substantial amount of
$\mathrm{DCO}^{+}$originates from temperatures above $30 \mathrm{~K}$ in the inner 70 au of the disk.

In addition to the mismatch between the relative levels of 2-1 and 4-3 emission, the B1a model underpredicts the $\mathrm{DCO}^{+}$ emission in the outer disk. We explore whether this mismatch can be explained by a second $\mathrm{DCO}^{+}$component around the dust edge, where UV photons may penetrate deeper into the disk, bringing excess cold $\mathrm{CO}$ into the gas phase. We modify B1a such that exterior to 60 au (the pebble disk edge is around $70 \mathrm{au}$ ) $\mathrm{DCO}^{+}$is present at $<27 \mathrm{~K}$, while interior to $60 \mathrm{au}$, the B1a model boundaries of $20<T<27 \mathrm{~K}$ still apply. This results in a more correct shape of the radial profile and may also explain the presence of a double-peaked $\mathrm{DCO}^{+}$radial profile. We emphasize that neither the $\mathrm{B} 1 \mathrm{a} / \mathrm{b}$ or $\mathrm{B} 2$ models correctly 
predict the 4-3/2-1 line ratios in the inner disks for $\mathrm{DCO}^{+}$and DCN. Since the B1 and B2 temperature boundaries simulate a cold emission layer, this mismatch suggests that neither molecule is mainly present in the cold midplane.

\section{Discussion}

\subsection{DCN and $\mathrm{DCO}^{+}$Radial and Vertical Structures}

In the inner regions of the TW Hya disk, both DCN and $\mathrm{DCO}^{+}$emission (and column densities) increase rapidly with increasing radius starting around $20-25 \mathrm{au}$, corresponding to midplane temperatures of $27-30 \mathrm{~K}$. In the absence of multiline observations, this observation would have been in line with expectations, since if $\mathrm{DCO}^{+}$forms from reactions between the cold gas tracer $\mathrm{H}_{2} \mathrm{D}^{+}$and $\mathrm{CO}$, $\mathrm{DCO}^{+}$should be most abundant in the midplane just interior to the $\mathrm{CO}$ snowline (e.g., Mathews et al. 2013; Aikawa et al. 2018). However, the inferred warm $\mathrm{DCO}^{+}$excitation temperature at 25 au shows that $\mathrm{DCO}^{+}$(and DCN) cannot be primarily emitting from the midplane. Instead the measured excitation temperature of $\sim 40 \mathrm{~K}$ at $25-60$ au places a substantial amount of the $\mathrm{DCO}^{+}$in an elevated disk layer. This discovery could point to a relatively inefficient lowtemperature $\mathrm{H}_{2} \mathrm{D}^{+}$fractionation chemistry and an efficient deuterium enrichment through reactions with, e.g., $\mathrm{CH}_{2} \mathrm{D}^{+}$. The latter is expected to proceed at higher temperatures than the $\mathrm{H}_{2} \mathrm{D}^{+}$chemistry and may therefore be consistent with a 40 K excitation temperature (Wootten 1987; Parise et al. 2009; Favre et al. 2015; Roueff et al. 2015).

This proposed scenario presents a new puzzle, however, which is that we do not observe much $\mathrm{DCN}$ and $\mathrm{DCO}^{+}$in the innermost midplane region. If we are observing a deuterium fractionation chemistry that is active at $\sim 40 \mathrm{~K}$, we would naively expect abundant $\mathrm{DCO}^{+}$and $\mathrm{DCN}$ in the inner disk midplane regions that correspond to this temperature, roughly $\sim 10-20$ au. Exterior to the $\mathrm{DCO}^{+}$and DCN cavity, a possible solution to the observed emission pattern is that there are two $\mathrm{DCO}^{+}$and DCN production zones, cold and warm respectively, which when observed toward a face-on disk will masquerade as the lukewarm emission layer we observe. Indeed the A1 model in Section 4 shows that this is a possibility from an excitation point of view. Whether this scenario is chemically plausible is less clear, and we still need an explanation as to why $\mathrm{DCO}^{+}$in TW Hya appears much warmer than in, e.g., the HD 162936 disk (Flaherty et al. 2017). In the inner disk the lack of $\mathrm{DCO}^{+}$and $\mathrm{DCN}$ may in part be explained by continuum blocking out some fraction of the molecular emission, making the hole seem deeper than it really is. However, as discussed previously, we do not think that it is likely that continuum opacity alone is responsible for the central cavity. Instead we suggest that the lack of $\mathrm{DCO}^{+}$ and $\mathrm{DCN}$ in the inner disk and the elevated temperature, and therefore elevated location of $\mathrm{DCO}^{+}$exterior to $25 \mathrm{au}$, together point toward a relatively inefficient $\mathrm{DCO}^{+}$and $\mathrm{DCN}$ production in the disk midplane at all disk radii.

One possible explanation for the lower than expected $\mathrm{DCO}^{+}$ midplane abundances is that $\mathrm{CO}$ is depleted in the disk far beyond the $\mathrm{CO}$ freeze-out zone due to, e.g., chemical processing and diffusion (e.g., Meijerink et al. 2009; Xu et al. 2017; Schwarz et al. 2018). If CO depletion begins at $40 \mathrm{~K}$ instead of $25 \mathrm{~K}$, this would explain why $\mathrm{DCO}^{+}$production is low both in the inner disk midplane and close to the $\mathrm{CO}$ snow surface in the outer disk. This explanation is supported by observations that show that the TW Hya disk is very CO-depleted throughout the disk molecular layers including in the inner disk (Favre et al. 2013; Kama et al. 2016; Schwarz et al. 2016; Zhang et al. 2019). CO depletion could also diminish DCN formation in the same locations, since $\mathrm{CO}$ depletion from the gas would result in a depletion of the overall carbon reservoir that controls DCN and HCN production. However, there is both observational (HilyBlant et al. 2010) and theoretical (Long et al. 2020) evidence that $\mathrm{HCN}$ (and by extension DCN) is not very sensitive to $\mathrm{CO}$ depletion, and this idea should therefore be considered highly speculative. DCN may be present at elevated disk layers simply because it mainly forms through the $\mathrm{CH}_{2} \mathrm{D}^{+}$pathway.

If $\mathrm{CO}$ depletion controls where in a disk there is an active deuterium fractionation chemistry, the TW Hya results may be far from universal. $\mathrm{CO}$ depletion through either chemistry or diffusive flows is expected to become more severe with disk age, and TW Hya has an unusually old disk. By contrast, we may expect to find colder and more midplane oriented $\mathrm{DCO}^{+}$ in disks that are less depleted in CO. Interestingly in the disk around Herbig Ae star HD 163296, which has been shown to be much less depleted in CO than TW Hya (Zhang et al. 2019), the $\mathrm{DCO}^{+}$excitation temperature is low $(<20 \mathrm{~K}$; Flaherty et al. 2017). By contrast, most $\mathrm{DCO}^{+}$in the disk around the Herbig Ae star HD 169142, appears to be warm (Carney et al. 2018). Additional observations toward a sample of young and old $\mathrm{T}$ Tauri and Herbig Ae disks would be key to resolve if the $\mathrm{DCO}^{+}$(and DCN) chemistry migrates to elevated disk layers over time, and if there are systematic differences between $\mathrm{T}$ Tauri and Herbig Ae disks.

A second possible explanation for the inferred low levels of $\mathrm{DCO}^{+}$in the TW Hya disk midplane is that the disk midplane regions are chemically quenched due to a lack of ionization. Close to the $\mathrm{CO}$ snow surface, there may be too little ionizing radiation to drive a $\mathrm{H}_{2} \mathrm{D}^{+}$- or $\mathrm{CH}_{2} \mathrm{D}^{+}$-mediated chemistry, and the measured excitation temperature of $\mathrm{DCO}^{+}$may reflect the coldest disk layer where an ion-molecule mediated deuterium fractionation chemistry is efficient. TW Hya has been inferred to have a low level of ionization throughout most of the disk (Cleeves et al. 2015), which supports this scenario. If this is the primary explanation for the lack of $\mathrm{DCO}^{+}$and $\mathrm{DCN}$ in the midplane, we would expect to see a decreasing $\mathrm{DCO}^{+}$ temperature with radius, since ionization should increase in the outer, more tenuous disk regions. Indeed such a decrease is detected exterior to $60 \mathrm{au}$, but we note that there are also other possible reasons for this decrease in $\mathrm{DCO}^{+}$temperature, including the release of cold $\mathrm{CO}$ into the gas phase through photodesorption (Öberg et al. 2015). Additional high-resolution $\mathrm{DCO}^{+}$and DCN observations toward disks with estimated ionization levels are needed to test this hypothesis.

Finally, we note that it is an open question whether we should also expect $\mathrm{DCO}^{+}$and $\mathrm{DCN}$ in the warm disk atmosphere in the inner disk. Favre et al. (2015) predict that $\mathrm{DCO}^{+}$should form abundantly in this disk region, while Aikawa et al. (2018) have come to a different conclusion. More theoretical work is needed to resolve this, but in the meantime we note that in the case of TW Hya, there is no evidence that the inner disk atmosphere is an important source of deuterated molecules.

The above discussion is relevant for both $\mathrm{DCN}$ and $\mathrm{DCO}^{+}$. We now proceed with exploring reasons for observed differences between the two molecules. First, based on emission profiles and toy models, the DCN cavity is somewhat 
smaller and/or less empty than the $\mathrm{DCO}^{+}$one. This suggests that there is at least one warm deuterium fractionation pathway that mainly affects DCN. There is tentative evidence for this warmer formation channel being important for DCN throughout the disk, since the disk-averaged DCN excitation temperature appears higher than that of $\mathrm{DCO}^{+}$. This, however, needs to be revisited with deeper DCN 2-1 observations.

In the outer disk of TW Hya, the $\mathrm{DCN}$ and $\mathrm{DCO}^{+}$radial profiles also diverge. While DCN presents a halo exterior to the pebble disk emission, $\mathrm{DCO}^{+}$has a much more substantial second emission component close to the edge of the pebble disk. Similar differences have been seen in other disks, most notably toward IM Lup and HD 163296 (Öberg et al. 2015; Huang et al. 2017; Salinas et al. 2017). The origin of a $\mathrm{DCO}^{+}$peak at the pebble disk edge is likely a result of increased penetration of UV radiation in the less shielded outer disk regions, which results in CO sublimation due to either a thermal inversion (Cleeves 2016) or enhanced CO ice photodesorption (Öberg et al. 2015; Huang et al. 2016; Aikawa et al. 2018). Cold, CO-rich gas constitutes an ideal environment for $\mathrm{DCO}^{+}$formation through the $\mathrm{H}_{2} \mathrm{D}^{+}$ channel. DCN clearly requires something in addition to this to form efficiently, though the presence of the DCN halo suggests that a small amount of DCN also forms under these conditions. One possible avenue to test whether the $\mathrm{DCO}^{+}$and $\mathrm{DCN}$ in the outer disk originate with $\mathrm{H}_{2} \mathrm{D}^{+}$would be to add observations of $\mathrm{N}_{2} \mathrm{D}^{+} . \mathrm{N}_{2} \mathrm{D}^{+}$only forms through reactions with $\mathrm{H}_{2} \mathrm{D}^{+}$and could therefore be used to map out where this pathway is active (see, e.g., Pagani et al. 2007; Salinas et al. 2017; Aikawa et al. 2018; Caselli et al. 2019). An important complication is that $\mathrm{N}_{2} \mathrm{D}^{+}$is only expected where there is substantial $\mathrm{CO}$ freeze-out, and not seeing $\mathrm{N}_{2} \mathrm{D}^{+}$can therefore not be used to rule out the $\mathrm{H}_{2} \mathrm{D}^{+}$ pathway.

In summary, there is evidence for active deuterium fractionation chemistry in the TW Hya disk. However, much of the observed emission from $\mathrm{DCO}^{+}$and $\mathrm{DCN}$ appears to originate well above the $\mathrm{CO}$ snow surface, and some process, perhaps $\mathrm{CO}$ depletion or low disk midplane ionization, may be limiting the efficiency of the cold pathway in the lower disk layers and in the inner disk midplane.

\subsection{DCN and DCO ${ }^{+}$Column Densities and Abundances}

$\mathrm{DCO}^{+}$and $\mathrm{DCN}$ have been observed and characterized toward TW Hya in a number of earlier studies. For reference, we extracted disk-averaged column densities of $\mathrm{DCO}^{+}$and DCN of 3.9 and $2.6 \times 10^{12} \mathrm{~cm}^{-2}$, respectively, and a $\mathrm{DCO}^{+}$ peak column density of $\sim 7 \times 10^{12} \mathrm{~cm}^{-2}$. Both are substantially higher compared to values derived from single-dish observations of 3 and $<0.4 \times 10^{11} \mathrm{~cm}^{-2}$ for $\mathrm{DCO}^{+}$and $\mathrm{DCN}$, respectively (van Dishoeck et al. 2003; Thi et al. 2004). This difference can likely be explained by beam dilution in the single-dish observations. The large difference in DCN and $\mathrm{DCO}^{+}$column densities inferred from single-dish observations is probably a beam dilution effect as well, since we find DCN to be more compact than $\mathrm{DCO}^{+}$.

$\mathrm{DCO}^{+}$and $\mathrm{DCN}$ have also been marginally resolved by Qi et al. (2008) and Öberg et al. (2012), and these observations were used to derive the radial column density profiles. Qi et al. (2008) found a peak column density of $\sim 4 \times 10^{12} \mathrm{~cm}^{-2}$, close to our measurement. By contrast the estimates of the DCN disk-averaged column density in Qi et al. (2008) and Öberg et al. (2012) are an order of magnitude lower than we find here. Some of this may be explained by beam averaging, since the synthesized beam in Öberg et al. (2012) was larger than the resolved DCN emitting region. The remaining difference can probably be accounted for by a different disk temperature structure and DCN emitting layer assumptions.

$\mathrm{DCO}^{+}$column densities and abundances have also been estimated toward a handful of other disks and the results are remarkably similar to those we find toward TW Hya; Teague et al. (2015) and Qi et al. (2015) found $\mathrm{DCO}^{+}$column densiyies toward DM Tau and HD 163296 of $\sim 10^{12} \mathrm{~cm}^{-2}$, and Carney et al. (2018) and Salinas et al. (2018) found $\mathrm{DCO}^{+}$abundances with respect to hydrogen toward HD 169142 and HD 163296 of $0.9-1.5 \times 10^{-12}$ and $2-6 \times 10^{-12}$, respectively. The consistent $\mathrm{DCO}^{+}$column densities and abundances toward this sample of four disks is difficult to interpret, since the $\mathrm{DCO}^{+}$ emitting layer appears quite different in, e.g., TW Hya and HD 163296. Finally, Salinas et al. (2017) also estimated the DCN abundance in the HD 163296 disk, and found $\sim 10^{-12}$ per hydrogen nuclei, which is again consistent with TW Hya.

\subsection{Model Comparison}

Model predictions of $\mathrm{DCN}$ and $\mathrm{DCO}^{+}$column density profiles, abundances, and emitting layers go back to the early 2000s. In a majority of models, $\mathrm{DCO}^{+}$column densities across disks are $\sim 10^{12} \mathrm{~cm}^{-2}$, in good agreement with the TW Hya findings (Aikawa \& Herbst 2001; Willacy 2007; Aikawa et al. 2018), but as discussed below this may be a coincidence since the $\mathrm{DCO}^{+}$distributions in TW Hya and in fiducial model disks appear quite different. A notable exception is Favre et al. (2015), who predicted substantially higher column densities due to efficient warm $\mathrm{DCO}^{+}$formation. In contrast to our findings, DCN is predicted to be at least an order of magnitude less abundant than $\mathrm{DCO}^{+}$in most models (Aikawa \& Herbst 2001; Aikawa et al. 2002; Willacy 2007; Favre et al. 2015). The one exception is one model in Willacy (2007), which predicts similarly high $\mathrm{DCN}$ and $\mathrm{DCO}^{+}$column densities. This model includes efficient ice photodesorption, which both increases the overall gas-phase carbon reservoir, and desorbs some of the DCN that forms through grain surface chemistry in their model. We speculate that the difference between models and observations with regard to the relative $\mathrm{DCO}^{+}$and $\mathrm{DCN}$ abundances may be due to a high $\mathrm{C} / \mathrm{O}$ ratio in the TW Hya disk, which would enhance both $\mathrm{HCN}$ production and the importance of the $\mathrm{CH}_{2} \mathrm{D}^{+}$fractionation pathway.

Models also predict shapes of radial profiles. In most models, $\mathrm{DCO}^{+}$displays a prominent inner hole, while DCN does not (Aikawa \& Herbst 2001; Willacy 2007; Willacy \& Woods 2009; Aikawa et al. 2018). This results in different $\mathrm{DCO}^{+}$and $\mathrm{DCN}$ radial profiles across the disk, in contrast to what is observed in both TW Hya and HD 163296, where DCN and $\mathrm{DCO}^{+}$appear to coincide at intermediate disk radii. This mismatch between models and observations suggests that the relative contributions of cold and warm deuterium fractionation pathways to $\mathrm{DCO}^{+}$and DCN remain to be fully worked out in disks.

\section{Conclusions}

1. $\mathrm{DCO}^{+}$and DCN 4-3, 3-2, and 2-1 have been observed at a spatial resolution of 0 !" $2-0$ !. 4 toward the TW Hya disk. DCN presents a single narrow ring and a diffuse halo in all transitions, while $\mathrm{DCO}^{+}$presents a broader ring that breaks up into multiple components at high spatial resolution. The inner edges of the radial profiles of 
all $\mathrm{DCN}$ and $\mathrm{DCO}^{+}$transitions are similar, but not identical.

2. Disk-averaged rotational diagrams show that $\mathrm{DCO}^{+}$is present at lukewarm temperatures, just under $40 \mathrm{~K}$, throughout most of the TW Hya disk, while DCN is likely warmer. The disk-averaged column densities are $\sim 4$ and $3 \times 10^{12}$ $\mathrm{cm}^{-2}$ for $\mathrm{DCO}^{+}$and $\mathrm{DCN}$, respectively.

3. Based on a series of parametric toy models, DCN emission is well fit by an inner 25 au (not completely empty) hole and a constant abundance outside of 25 au at temperatures $>20 \mathrm{~K}$. By contrast $\mathrm{DCO}^{+}$cannot be fit by any single constant abundance distribution, but requires an abundance model that takes into account the presence of a second cold reservoir of $\mathrm{DCO}^{+}$in the outer disk.

4. $\mathrm{DCN}$ and $\mathrm{DCO}^{+}$production may share a formation pathway in the inner disk, where the radial profiles of the two molecules resemble one another, i.e., both molecules become abundant at elevated disk layers at $\sim 25 \mathrm{au}$. In these lukewarm layers hydrocarbon-mediated deuterium fractionation should be efficient, though we cannot exclude that the $\mathrm{H}_{2} \mathrm{D}^{+}$pathway contributes as well. DCN presents a small shoulder interior to the main radial peak, which suggests that there is a second warmer deuterium fractionation pathway that results in DCN, but not $\mathrm{DCO}^{+}$, production. In the outer disk, exterior to the pebble disk, $\mathrm{DCO}^{+}$is much more abundant than $\mathrm{DCN}$ and also seems to exist at lower temperatures indicative of a cold, $\mathrm{H}_{2} \mathrm{D}^{+}$-mediated deuterium chemistry.

5. Deuterium fractionation chemistry is generally thought of as being a low-temperature process. In the case of TW Hya, deuterated molecules instead appear to mainly emit from an intermediate temperature disk layer, which suggests that either $\mathrm{CO}$ removal from the gas phase or a lack of ionizing radiation has diminished the deuterium chemistry in the disk midplane.

Deuterium fractionation in disks is complex and multifaceted. Multiline observations are key to constrain excitation temperatures of abundant deuterated molecules, and further, to determine under which disk conditions they form. Ideally this should be combined with direct measurements of emission layer heights in samples of moderately inclined disks to obtain conclusive data on where and through which processes different molecules can become fractionated in deuterium. We note that TW Hya is an old, and extremely CO-depleted disk, and it will be very interesting to explore whether younger disks display a similarly distributed deuterium fractionation chemistry, or whether they enable deuterium fractionation closer to the planet-forming midplane.
This paper makes use of the following ALMA data: ADS/JAO. ALMA\#2016.1.00311.S and ADS/JAO.ALMA\#2016.1.00440. S. ALMA is a partnership of ESO (representing its member states), NSF (USA) and NINS (Japan), together with NRC (Canada), MOST and ASIAA (Taiwan), and KASI (Republic of Korea), in cooperation with the Republic of Chile. The Joint ALMA Observatory is operated by ESO, AUI/NRAO, and NAOJ. This work is supported by the National Radio Astronomy Observatory (NRAO). NRAO is a facility of the National Science Foundation operated under cooperative agreement by Associated Universities, Inc. This work was supported by an award from the Simons Foundation (SCOL \# 321183, KÖ). K.I.Ö. also gratefully acknowledges support from the David and Lucille Packard Foundation. L.I.C. gratefully acknowledges support from the David and Lucille Packard Foundation and the Johnson \& Johnson WISTEM2D Award. J.B.B. acknowledges support from NASA through the NASA Hubble Fellowship grant \#HST-HF251429.001-A awarded by the Space Telescope Science Institute, which is operated by the Association of Universities for Research in Astronomy, Incorporated, under NASA contract NAS5-26555. J.T.v.S. and M.R.H. are supported by the Dutch Astrochemistry II program of the Netherlands Organization for Scientific Research (648.000.025). J.H. acknowledges that support for this work was provided by NASA through the NASA Hubble Fellowship grant \#HST-HF2-51460.001-A awarded by the Space Telescope Science Institute, which is operated by the Association of Universities for Research in Astronomy, Inc., for NASA, under contract NAS5-26555. C.W acknowledges financial support from the University of Leeds and from the Science and Technology Facilities Council (grant Nos. ST/R000549/1 and ST/T000287/ 1). J.K.C. acknowledges support from the National Science Foundation Graduate Research Fellowship under Grant No. DGE 1256260 and the National Aeronautics and Space Administration FINESST grant, under Grant No. 80NSSC19K1534.

Facility: ALMA.

Software: CASA (McMullin et al. 2007), Astropy (Astropy Collaboration et al. 2013, 2018), RADMC-3D (Dullemond 2012).

\section{Appendix Channel Maps}

Figures 9 and 10 display the channel maps of the DCN 2-1, 3-2, and 4-3 emission, and the $\mathrm{DCO}^{+}$2-1, 3-2, and 4-3 emission, respectively. These were used to create the moment maps, radial profiles, and spectra shown in the main body of the paper. 


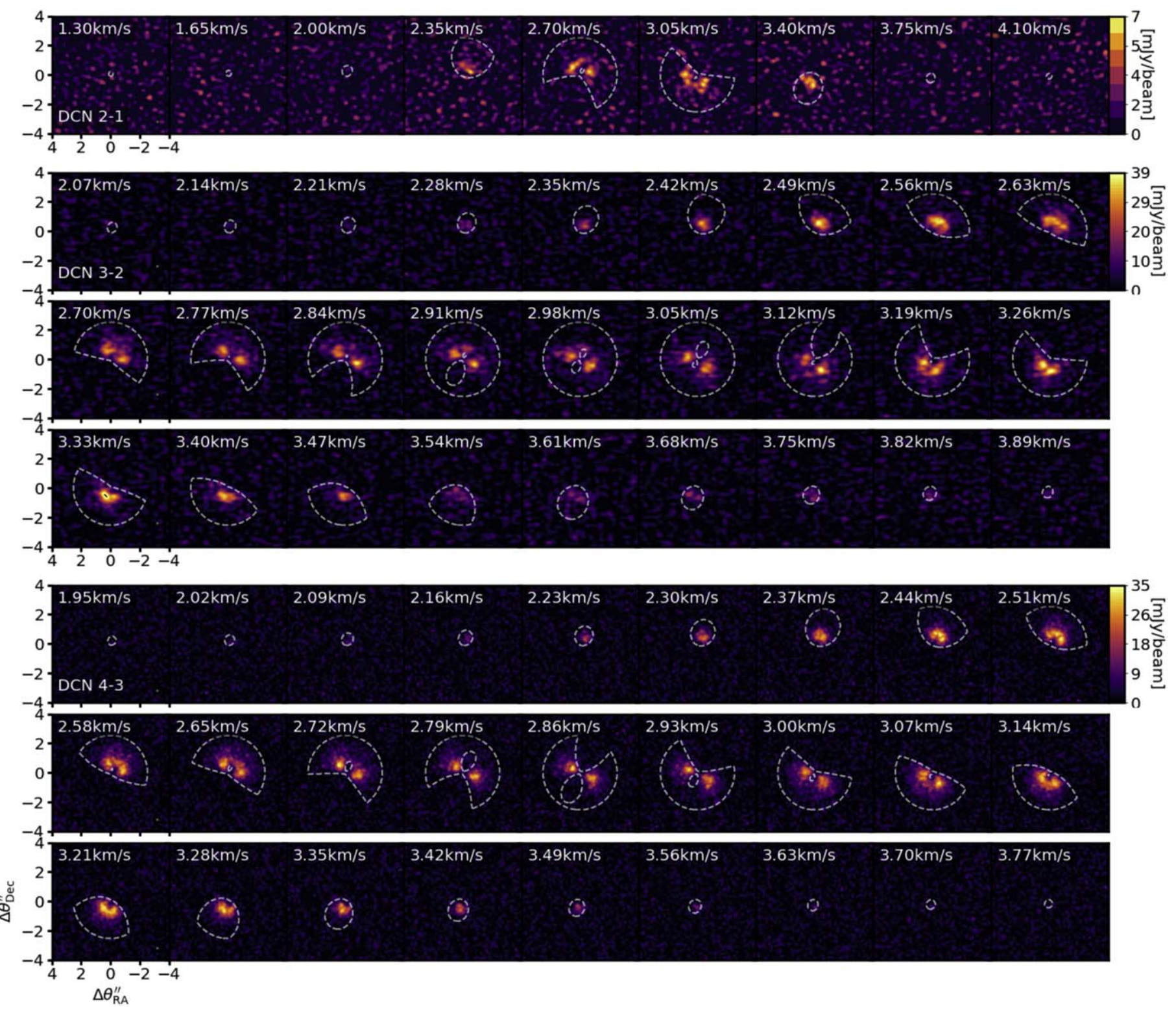

Figure 9. DCN channel maps using the fiducial imaging parameters. The Keplerian mask used to extract spectra is overplotted. 

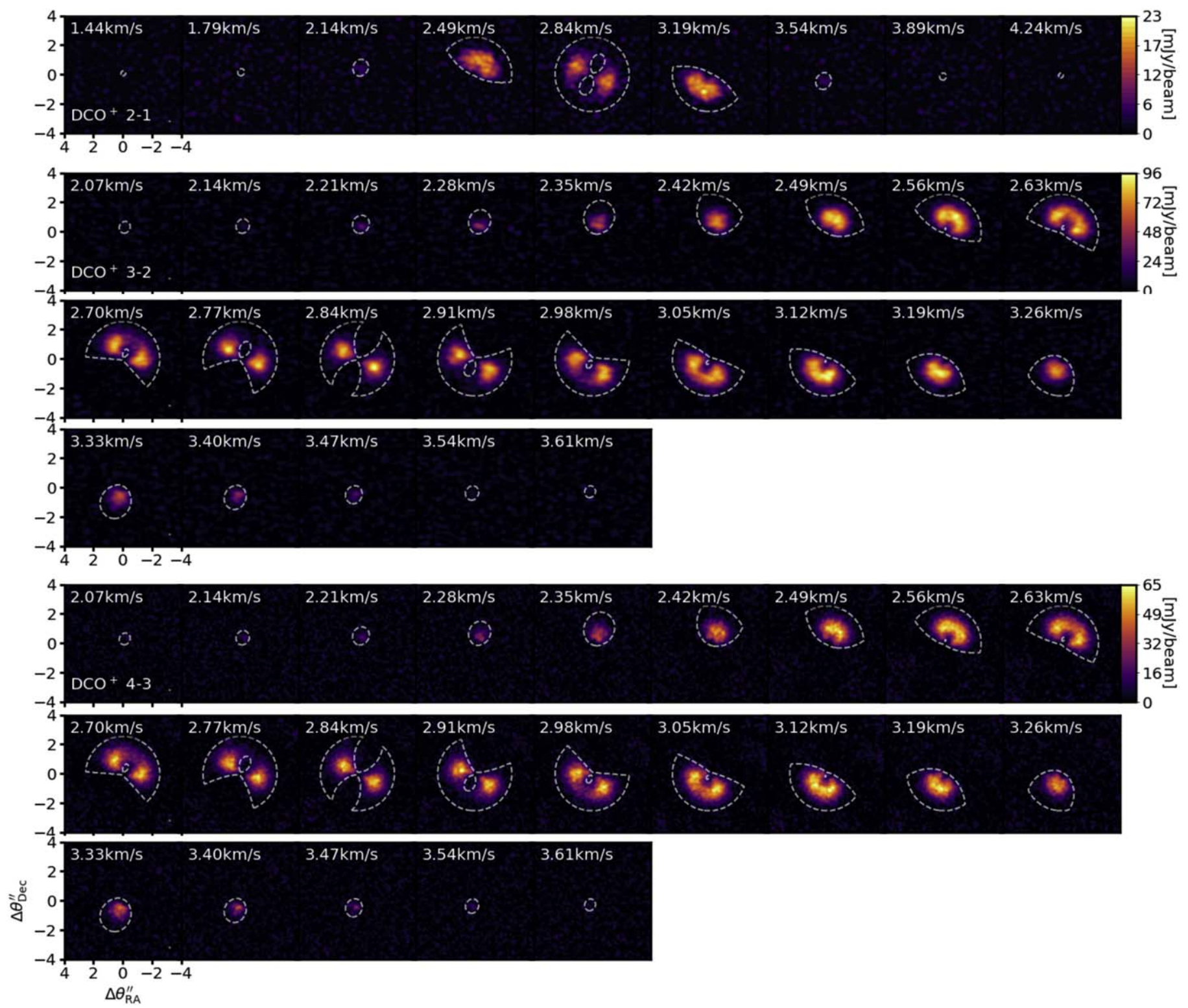

Figure 10. $\mathrm{DCO}^{+}$channel maps using the fiducial imaging parameters. The Keplerian mask used to extract spectra is overplotted.

\section{ORCID iDs}

Karin I. Öberg (1) https://orcid.org/0000-0001-8798-1347 L. Ilsedore Cleeves (ib https://orcid.org/0000-0003-2076-8001 Jennifer B. Bergner (1) https://orcid.org/0000-0002-8716-0482 Richard Teague (ib https://orcid.org/0000-0003-1534-5186 Jane Huang (1) https://orcid.org/0000-0001-6947-6072 Ryan A. Loomis (10) https://orcid.org/0000-0002-8932-1219 Edwin A. Bergin (1) https://orcid.org/0000-0003-4179-6394 Geoffrey A. Blake (1) https://orcid.org/0000-0003-0787-1610 Jenny Calahan 는 https://orcid.org/0000-0002-0150-0125 Michiel R. Hogerheijde (1) https://orcid.org/0000-0001-5217-537X Mihkel Kama @ https://orcid.org/0000-0003-0065-7267 Jeroen Terwisscha van Scheltinga (10 https://orcid.org/00000002-3800-9639

Chunhua Qi i https://orcid.org/0000-0001-8642-1786 Ewine van Dishoeck (1) https://orcid.org/0000-00017591-1907

Catherine Walsh (ํ) https://orcid.org/0000-0001-6078-786X David J. Wilner (i) https://orcid.org/0000-0003-1526-7587

\section{References}

Aikawa, Y., Furuya, K., Hincelin, U., \& Herbst, E. 2018, ApJ, 855, 119 Aikawa, Y., \& Herbst, E. 1999, A\&A, 351, 233

Aikawa, Y., \& Herbst, E. 2001, A\&A, 371, 1107

Aikawa, Y., van Zadelhoff, G. J., van Dishoeck, E. F., \& Herbst, E. 2002, A\&A, 386, 622

Alexander, C. M. O. D., Cody, G. D., De Gregorio, B. T., Nittler, L. R., \& Stroud, R. M. 2017, ChEG, 77, 227

Altwegg, K., Balsiger, H., Bar-Nun, A., et al. 2015, Sci, 347, 1261952 Altwegg, K., Balsiger, H., \& Fuselier, S. A. 2019, ARA\&A, 57, 113

Andrews, S. M., Wilner, D. J., Hughes, A. M., et al. 2012, ApJ, 744, 162 Andrews, S. M., Wilner, D. J., Zhu, Z., et al. 2016, ApJL, 820, L40

Astropy Collaboration, Robitaille, T. P., Tollerud, E. J., et al. 2013, A\&A, 558, A33

Astropy Collaboration, Price-Whelan, A. M., Sipőcz, B. M., et al. 2018, AJ, 156,123

Bergin, E. A., Cleeves, L. I., Gorti, U., et al. 2013, Natur, 493, 644

Carney, M. T., Fedele, D., Hogerheijde, M. R., et al. 2018, A\&A, 614, A106 Caselli, P., Sipilä, O., \& Harju, J. 2019, RSPTA, 377, 20180401

Ceccarelli, C., Caselli, P., Bockelée-Morvan, D., et al. 2014, in Protostars and Planets VI, ed. H. Beuther et al. (Tucson, AZ: Univ. Arizona Press), 914 Cleeves, L. I. 2016, ApJL, 816, L21

Cleeves, L. I., Bergin, E. A., Alexander, C. M. O., et al. 2014, Sci, 345, 1590 
Cleeves, L. I., Bergin, E. A., Qi, C., Adams, F. C., \& Öberg, K. I. 2015, ApJ, 799, 204

Dartois, E., Dutrey, A., \& Guilloteau, S. 2003, A\&A, 399, 773

Dullemond, C. P. 2012, RADMC-3D: A Multi-Purpose Radiative Transfer Tool Astrophysics Source Code Library, ascl:1202.015

Dutrey, A., Guilloteau, S., Duvert, G., et al. 1996, A\&A, 309, 493

Favre, C., Bergin, E. A., Cleeves, L. I., et al. 2015, ApJL, 802, L23

Favre, C., Cleeves, L. I., Bergin, E. A., Qi, C., \& Blake, G. A. 2013, ApJL, 776, L38

Flaherty, K. M., Hughes, A. M., Rose, S. C., et al. 2017, ApJ, 843, 150

Foreman-Mackey, D., Conley, A., Meierjurgen Farr, W., et al. 2013, Emcee: The MCMC Hammer, Astrophysics Source Code Library, ascl:1303.002

Fuente, A., Cernicharo, J., Agúndez, M., et al. 2010, A\&A, 524, A19

Furuya, K., Drozdovskaya, M. N., Visser, R., et al. 2017, A\&A, 599, A40

Guilloteau, S., Piétu, V., Dutrey, A., \& Guélin, M. 2006, A\&A, 448, L5

Hartogh, P., Lis, D. C., Bockelée-Morvan, D., et al. 2011, Natur, 478, $218 \mathrm{H}$

Hily-Blant, P., Walmsley, M., Pineau Des Forêts, G., \& Flower, D. 2010, A\&A, 513, A41

Högbom, J. A. 1974, A\&AS, 15, 417

Huang, J., Andrews, S. M., Cleeves, L. I., et al. 2018, ApJ, 852, 122

Huang, J., \& Öberg, K. I. 2015, ApJL, 809, L26

Huang, J., Öberg, K. I., \& Andrews, S. M. 2016, ApJL, 823, L18

Huang, J., Öberg, K. I., Qi, C., et al. 2017, ApJ, 835, 231

Kama, M., Bruderer, S., van Dishoeck, E. F., et al. 2016, A\&A, 592, A83

Long, F., Bosman, A. D., \& Cazzoletti, P. 2020, A\&A, submitted

Loomis, R. A., Cleeves, L. I., Öberg, K. I., et al. 2018a, ApJ, 859, 131

Loomis, R. A., Öberg, K. I., Andrews, S. M., et al. 2018b, AJ, 155, 182

Loomis, R. A., Öberg, K. I., Andrews, S. M., et al. 2020, ApJ, 893, 101

Mathews, G. S., Klaassen, P. D., Juhász, A., et al. 2013, A\&A, 557, A132

McMullin, J. P., Waters, B., Schiebel, D., Young, W., \& Golap, K. 2007, in ASP Conf. Ser., 376, Astronomical Data Analysis Software and Systems XVI, ed. R. A. Shaw et al. (San Francisco, CA: ASP), 127

Meijerink, R., Pontoppidan, K. M., Blake, G. A., Poelman, D. R., \& Dullemond, C. P. 2009, ApJ, 704, 1471

Millar, T. J., Bennett, A., Rawlings, J. M. C., Brown, P. D., \& Charnley, S. B. 1991, A\&AS, 87, 585
Müller, H. S. P., Schlöder, F., Stutzki, J., \& Winnewisser, G. 2005, JMoSt, 742,215

Mumma, M. J., \& Charnley, S. B. 2011, ARA\&A, 49, 471

Öberg, K. I., Furuya, K., Loomis, R., et al. 2015, ApJ, 810, 112

Öberg, K. I., Qi, C., Fogel, J. K. J., et al. 2010, ApJ, 720, 480

Öberg, K. I., Qi, C., Fogel, J. K. J., et al. 2011, ApJ, 734, 98

Öberg, K. I., Qi, C., Wilner, D. J., \& Hogerheijde, M. R. 2012, ApJ, 749, 162

Pagani, L., Bacmann, A., Cabrit, S., \& Vastel, C. 2007, A\&A, 467, 179

Parise, B., Leurini, S., Schilke, P., et al. 2009, A\&A, 508, 737

Pegues, J., Öberg, K. I., Bergner, J. B., et al. 2020, ApJ, 890, 142

Qi, C., Öberg, K. I., Andrews, S. M., et al. 2015, ApJ, 813, 128

Qi, C., Wilner, D. J., Aikawa, Y., Blake, G. A., \& Hogerheijde, M. R. 2008, ApJ, 681, 1396

Robert, F., \& Epstein, S. 1982, GeCoA, 46, 81

Roueff, E., Loison, J. C., \& Hickson, K. M. 2015, A\&A, 576, A99

Salinas, V. N., Hogerheijde, M. R., Mathews, G. S., et al. 2017, A\&A, 606, A 125

Salinas, V. N., Hogerheijde, M. R., Murillo, N. M., et al. 2018, A\&A, 616, A45

Schöier, F. L., van der Tak, F. F. S., van Dishoeck, E. F., \& Black, J. H. 2005 , A\&A, 432, 369

Schwarz, K. R., Bergin, E. A., Cleeves, L. I., et al. 2016, ApJ, 823, 91

Schwarz, K. R., Bergin, E. A., Cleeves, L. I., et al. 2018, ApJ, 856, 85

Teague, R., Semenov, D., Guilloteau, S., et al. 2015, A\&A, 574, A137

Thi, W., van Zadelhoff, G., \& van Dishoeck, E. F. 2004, A\&A, 425, 955

Turner, B. E. 2001, ApJS, 136, 579

van Dishoeck, E. F., Thi, W., \& van Zadelhoff, G. 2003, A\&A, 400, L1

Willacy, K. 2007, ApJ, 660, 441

Willacy, K., \& Woods, P. M. 2009, ApJ, 703, 479

Wootten, A. 1987, in Proc. IAU Symp. 120, Astrochemistry, ed. M. S. Vardya \& S. P. Tarafdar (Cham: Springer), 311

Xu, R., Bai, X.-N., \& Öberg, K. 2017, ApJ, 835, 162

Yang, L., Ciesla, F. J., \& Alexander, C. M. O. D. 2013, Icar, 226, 256

Zhang, K., Bergin, E. A., Blake, G. A., Cleeves, L. I., \& Schwarz, K. R. 2017, NatAs, 1, 0130

Zhang, K., Bergin, E. A., Schwarz, K., Krijt, S., \& Ciesla, F. 2019, ApJ, 883, 98 